\title{
Recurrent intracranial neurenteric cyst with malignant transformation: A case report and literature review
}

\author{
YANG YANG $^{1}, \mathrm{JINGYI} \mathrm{FANG}^{2}, \mathrm{DA} \mathrm{LI}^{1}, \mathrm{LIANG} \mathrm{WANG}^{1}, \mathrm{NAN} \mathrm{JI}^{1}$ and $\mathrm{JUNTING} \mathrm{ZHANG}^{1}$ \\ ${ }^{1}$ Department of Neurosurgery, Beijing Tiantan Hospital, Capital Medical University; ${ }^{2}$ Department of Neuropathology, \\ Beijing Neurosurgical Institute, Capital Medical University, Beijing 100050, P.R. China
}

Received February 27, 2015; Accepted January 6, 2016

DOI: $10.3892 / \mathrm{ol} .2016 .4386$

\begin{abstract}
Neurenteric cysts (NCs) are uncommon congenital cystic lesions. Malignantly transformed NCs are extremely rare. The present study reports the case of a recurrent foramen magnum NC with a malignant transformation and reviews 8 previously reported cases. A 58-year-old woman with a 5-month history of occasional headaches, dizziness and vomiting presented to the Beijing Tiantan Hospital (Beijing, China) with palsy of the left cranial IX and X nerves, decreased sensation in the limbs on the right side and an ataxic gait. Magnetic resonance imaging (MRI) scans revealed a cystic mass in the left side of the foramen magnum, positioned anteriolaterally to the medulla oblongata. Surgery using a left suboccipital retrosigmoid approach was performed. The lesion was partially resected and was histopathologically diagnosed as an NC with focal hyperplasia of the epithelial cells. Additional MRI scans that were performed 25 months later revealed a solid lesion posterior to the medullar oblongata. Therefore, surgery using a suboccipital midline approach was performed. The solid lesion was sub-totally removed and was histopathologically diagnosed as a well-differentiated papillary adenocarcinoma. At 1 month post-surgery, the patient's condition worsened, eventually resulting in mortality due to respiratory failure. Based on the findings of the present case and previous literature, strict criteria for malignant-transformed NCs were created. The location distribution of malignant-transformed NCs does
\end{abstract}

Correspondence to: Dr Junting Zhang, Department of Neurosurgery, Beijing Tiantan Hospital, Capital Medical University, 6 Tiantan Xili, Beijing 100050, P.R. China

E-mail: zhangjunting2003@aliyun.com

Abbreviations: NC, neurenteric cyst; MRI, magnetic resonance imaging; EMA, epithelial membrane antigen; CEA, carcinoembryonic antigen; TTF-1, thyroid transcription factor 1; GFAP, glial fibrillary acidic protein; Vim, vimentin

Key words: foramen magnum, neurenteric cyst, malignant transformation, tumor recurrence not coincide with the location distribution of all NCs, and malignantly transformed NCs usually occur in adult patients and present as atypical radiological features. Surgery is the optimal treatment for malignantly transformed NCs, and the effectiveness of adjuvant therapy requires additional studies.

\section{Introduction}

Neurenteric cysts (NCs) are uncommon congenital cystic lesions of presumed ectodermal origin that rarely appear in the cranio-spinal axis. They are characterized by cyst walls that are lined with a simple or pseudostratified, ciliated or non-ciliated, cuboidal or columnar epithelium, with basement membranes that resemble those of the respiratory or intestinal tract (1). The precise pathogenesis of NCs remains controversial $(1,2)$. NCs are usually located in the spinal subdural space, particularly in the lower cervical and upper thoracic regions (3). Intracranial NCs are relatively infrequently reported, compared with the incidence of intraspinal cases (4). To the best of our knowledge, no more than 100 cases of intracranial $\mathrm{NC}$ have been reported in the literature $(1,4,5)$.

Surgery is the primary treatment for NC, and gross total resection of the lesion with careful protection of the surrounding structures is recommended (6). In the majority of cases, this rare benign lesion carries a favorable prognosis following surgical intervention (6-8). However, in some cases the remnants of the NCs, which occur due to subtotal or partial resection caused by adhesion between the NCs and surrounding structures, may develop into tumor recurrences (6). Therefore, close follow-up with radiological examination is required, particularly for patients for whom total resection of the lesion was not achieved.

Compared with the incidence of recurrent $\mathrm{NCs}$, the malignant transformation of NCs is extremely rare. To the best of our knowledge, very few reports have described this unusual phenomenon (9-16). Therefore, the demographic characteristics, treatment and prognosis of this type of $\mathrm{NC}$ is not clear. The objective of the present study was to analyze the clinical, pathological and radiological characteristics of malignant-transformed NCs based on the data from the present case combined with previous literature. In addition, strict criteria for malignant-transformed NCs were defined. Written informed consent was obtained from the patient. 
A



C

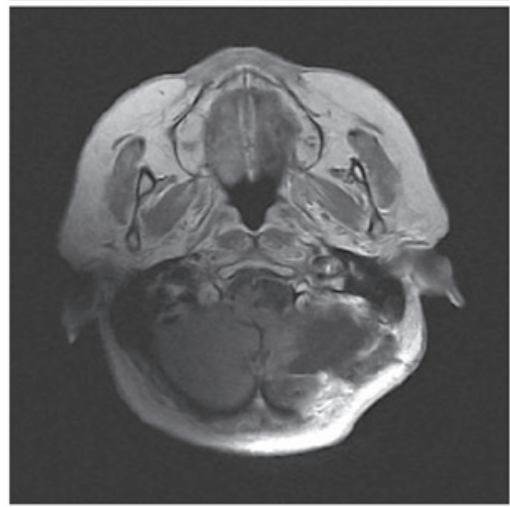

B

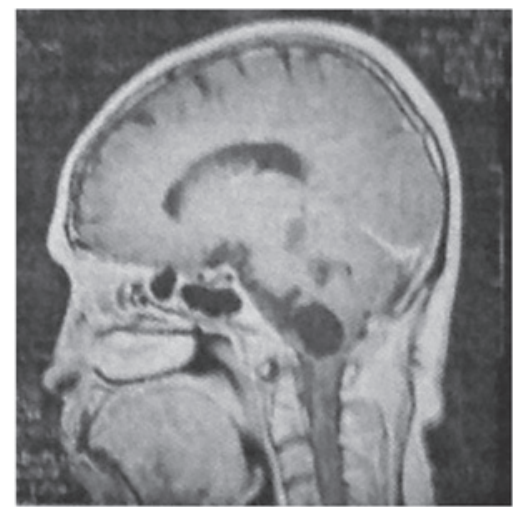

D



Figure 1. Pre- and post-operative MRI scans of the patient's second craniotomy. (A) Unenhanced axial T1-weighted and (B) Gd-enhanced sagittal T1-weighted MRI scans that were obtained prior to the second craniotomy revealed a cystic mass in the left side of the foramen magnum, anteriolateral to the medulla oblongata, which was compressed by the lesion. (C) Unenhanced axial T1-weighted and (D) Gd-enhanced sagittal T1-weighted MRI scans that were obtained subsequent to the second craniotomy revealed the restoration of the medulla oblongata following the partial resection of the lesion. MRI, magnetic resonance imaging; Gd, gadolinium.

\section{Case report}

On December 2, 2008, a 58-year-old woman presented to the Beijing Tiantan Hospital (Beijing, China), with a 5-month history of occasional headaches, dizziness and vomiting. The symptoms progressively worsened, and the patient began to suffer from intermittent dysphagia and an abnormal gait. A neurological examination revealed palsy of the left cranial IX and $\mathrm{X}$ nerves, decreased sensation in the limbs on the right side and an ataxic gait. The patient had undergone a left suboccipital retrosigmoid craniotomy to remove a lesion in the left cerebello-pontine angle 8 years previously in a local hospital. The lesion was pathologically diagnosed as an NC, as detailed radiological and histopathological data were not available. Magnetic resonance imaging (MRI; MAGNETOM Trio 3.0T superconducting magnetic resonance imager; Siemens AG, Munich, Germany) scans revealed a cystic mass in the left side of the foramen magnum that was located anteriolaterally to the medulla oblongata (Fig. 1). The patient underwent a second craniotomy using the left suboccipital retrosigmoid approach at the Beijing Tiantan Hospital. Following the removal of the cyst contents, the cyst walls were partially resected due to adhesion between the cyst wall and medulla oblongata. The pathological diagnosis was of an NC. The numbness in the right limbs and the left lower cranial nerve palsy improved without surgical morbidity.

At 22 months after the second craniotomy, the patient presented again with intermittent headaches and dizziness.
A radiological examination revealed supratentorial hydrocephalus. A right ventriculo-peritoneal shunt was performed, and subsequent improvement of the neurological symptoms was observed.

At 23 months after the second craniotomy, the patient presented with a severe headache, projectile vomiting, diplopia and limb fatigue. A neurological examination detected significant palsy of the left VI and VII cranial nerves, grade IV limb muscle strength and a positive Romberg test result, with the patient falling to the left.

Additional MRI scans demonstrated a solid lesion posterior to the medullar oblongata, with isointensity on the T1- and the T2-weighted images and homogeneous enhancement following the administration of gadolinium (Gd; Fig. 2). The lesion compressed the medullar oblongata. No brain parenchymal edema surrounding the lesion was noted. A total-body computed tomography scan was performed due to the possibility of an extracranial primary neoplasm, and no evidence of tumors in other organs or metastases was identified.

At 25 months after the second surgery in the Beijing Tiantan Hospital, the patient underwent a third craniotomy using a suboccipital midline approach to relieve the brainstem compression. Intra-operatively, the solid lesion was soft with a moderate blood supply. A subtotal resection of the tumor and a cyst evacuation were performed, as a total resection was not possible. Subsequent to the resection of the lesion, the infiltration of the medulla oblongata by the lesion was observed. 
A

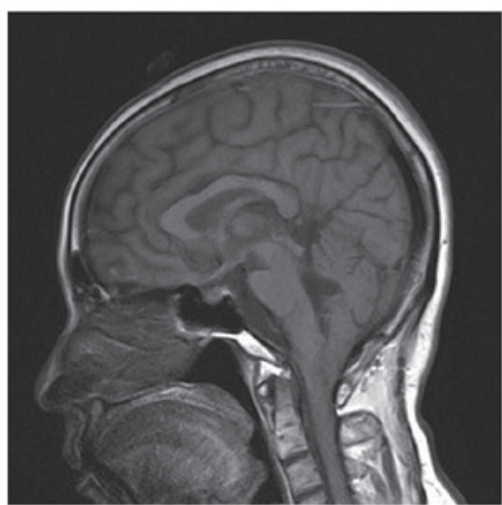

D



B

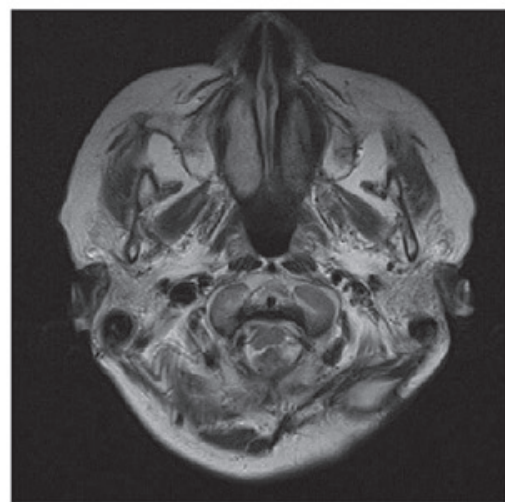

E

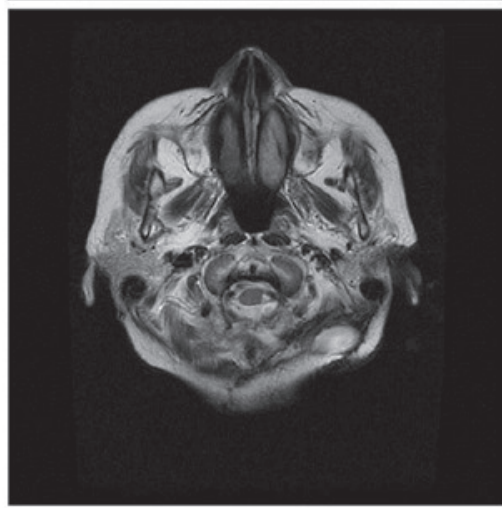

C

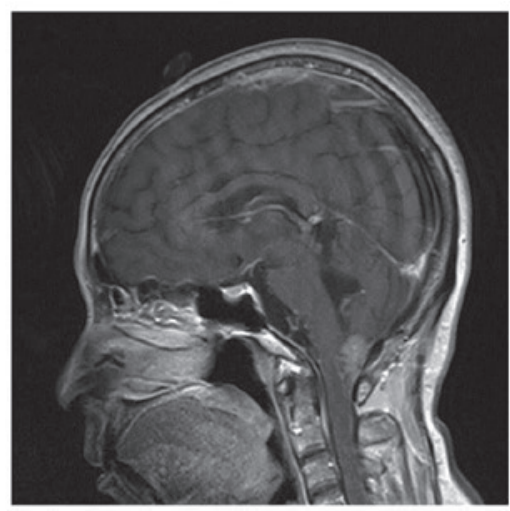

F



Figure 2. Pre- and post-operative MRI scans of the patient's third craniotomy. (A) Unenhanced sagittal T1-weighted,(B) axial T2-weighted and (C) Gd-enhanced sagittal T1-weighted MRI scans that were obtained prior to the third craniotomy revealed a solid lesion, posterior to the medullar oblongata, with iso-intensity on T1- and T2-weighted images and homogeneous enhancement following the administration of Gd. (D) Unenhanced sagittal T1-weighted, (E) axial T2-weighted and (F) Gd-enhanced sagittal T1-weighted MRI scans that were obtained subsequent to the third craniotomy revealed the disappearance of the lesion. MRI, magnetic resonance imaging; Gd, gadolinium.

A

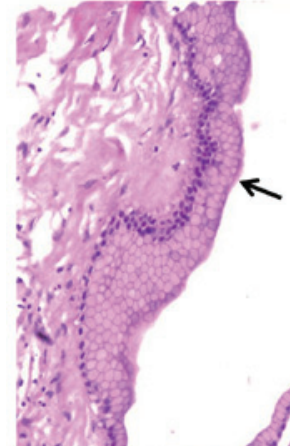

$\mathrm{C}$



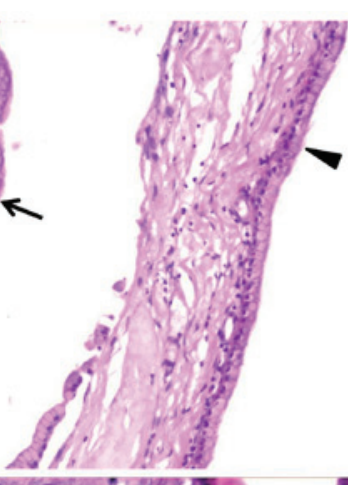

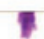

B

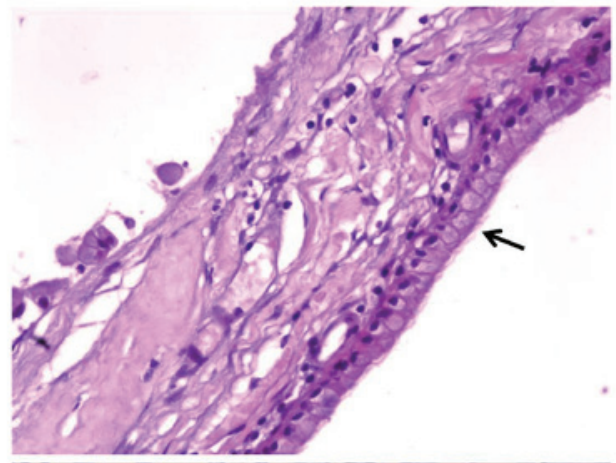

D

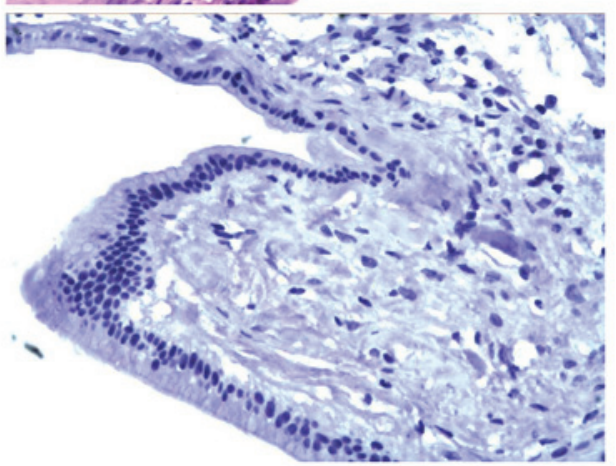

Figure 3. Histopathological and immunohistochemical features of the specimen obtained in the patient's second craniotomy. (A) The cyst wall was lined with single-layer or pseudostratified columnar and non-ciliated epithelial cells with goblet and mucin-producing cells (arrow). In certain regions, a single layer of ciliated epithelial cells was observed (arrowhead). Original magnification, x100. (B) Single-layer ciliated epithelial cells without a malignant component were observed. Original magnification, x200. (C) Focal hyperplasia of the epithelial cells, which is similar to the papillary structure, was observed in certain regions, and malignant characteristics, including nuclear pleomorphisms and frequent mitosis, were not detected. Original magnification, x200. (D) Immunohistochemical staining for the Ki-67 proliferation marker did not reveal any positively stained cells in the specimen. Original magnification, x200. 

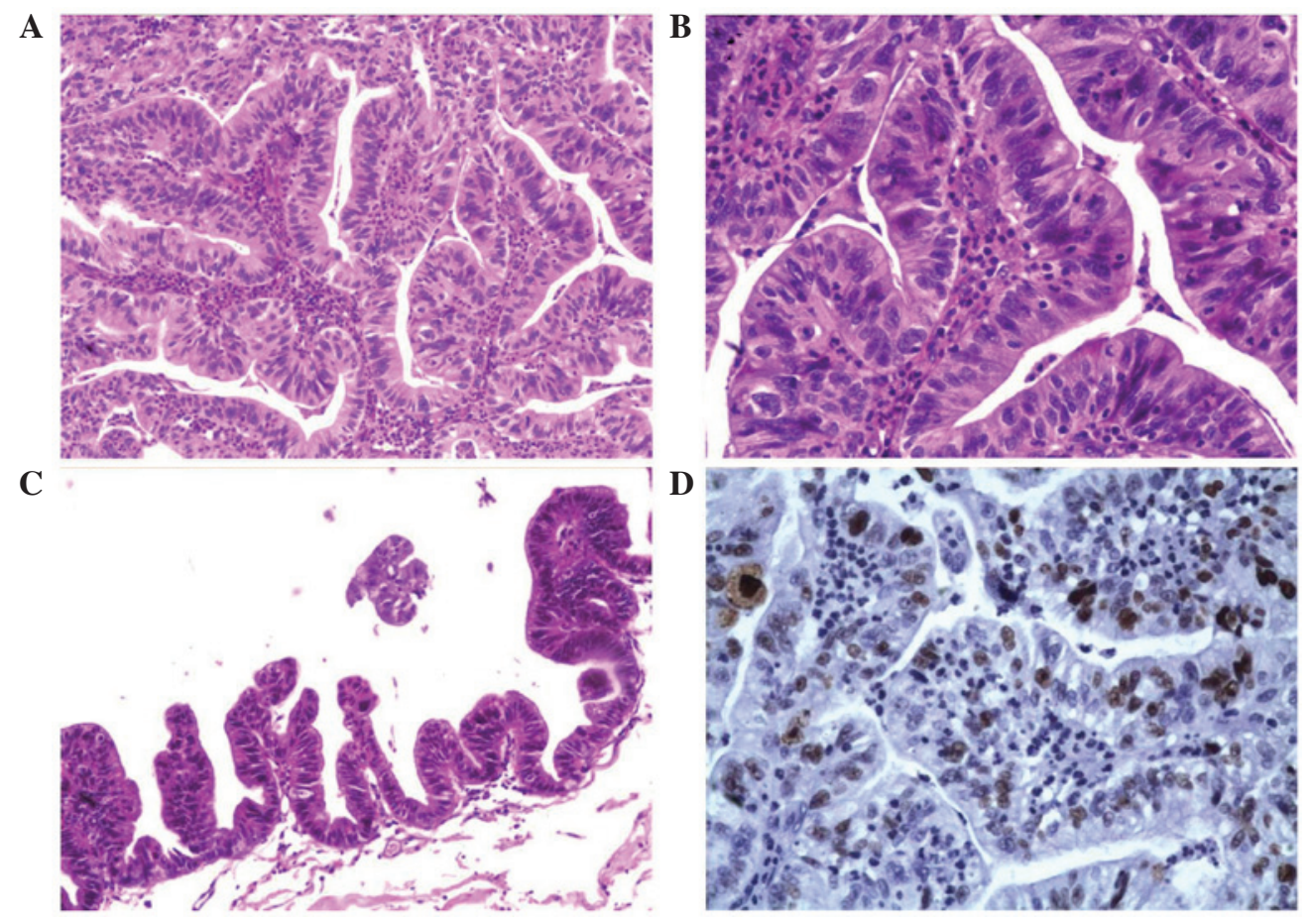

Figure 4. Histopathological and immunohistochemical features of the specimen obtained in the patient's third surgery. Well-formed papillae with prominent fibrovascular cores that were lined by malignant epithelium cells and contained enlarged hyperchromatic nuclei with a high nuclear to cytoplasmic ratio, nuclear abnormalities and mitotic figures were observed. Original magnification, (A) x100 and (B) x200. (C) In certain regions, frequent irregular folds of the dysplastic epithelial cells, which showed a tendency to form well-formed papillae structures, were observed. Original magnification, x100. (D) Immunohistochemical staining of the specimen revealed that the Ki-67 proliferation index had increased to 20-40\%. Original magnification, x 200 .

The upper limb muscle strength of the patient improved to grade $\mathrm{V}$ following the surgery, and the other symptoms were stabilized without novel neurological deficits. The patient was discharged at 15 days post-surgery with a good cough reflex. The follow-up revealed that after 1 month, the patient began to experience a decreased level of consciousness and ultimately succumbed to respiratory failure in hospital.

The specimens from the second and third surgeries were fixed in $10 \%$ formalin and embedded in paraffin. The $4-\mu \mathrm{m}$ thick sections were cut and stained with hematoxylin and eosin. Selected sections were also immunostained using the following primary antibodies: glial fibrillary acidic protein (GFAP; monoclonal mouse immunoglobulin (Ig) G anti-rat/chicken/human/pig; dilution, 1:100; catalog no., MA5-12023; Thermo Fisher Scientific, Inc., Waltham, MA, USA), epithelial membrane antigen (EMA; monoclonal Armenian hamster IgG anti-mouse/human; dilution, 1:50; catalog no., MA5-11202; Thermo Fisher Scientific, Inc.), carcinoembryonic antigen (CEA; monoclonal mouse IgG anti-human; dilution, 1:50; catalog no., MA5-15070 Thermo Fisher Scientific, Inc.), S-100 protein (monoclonal mouse IgG anti-human/rat/mouse/cattle; dilution, 1:50; catalog no., MA5-12966; Thermo Fisher Scientific, Inc.), thyroid transcription factor-1 (TTF-1; monoclonal mouse IgG anti-human/mouse/rat; dilution, 1:100; catalog no.,MA5-13961 Thermo Fisher Scientific, Inc.) and Ki-67 (MIB-1; monoclonal rabbit IgG anti-human; dilution, 1:50; catalog no., RM9106R1; Labvision, Fremont, CA, USA).

The histological examination of the entire specimen from the second surgery revealed that the cyst wall was lined with single-layer or pseudostratified columnar and non-ciliated epithelial cells, mixed with goblet and mucin-producing cells, but without malignant components (Fig. 3A). Single-layer ciliated epithelial cells were also observed in certain regions (Fig. 3A and B). Focal hyperplasia of the epithelial cells, which was similar to a papillary structure, was observed in certain regions (Fig. 3C); however, malignant characteristics, including nuclear pleomorphisms and frequent mitosis, were not observed. The immunohistological staining revealed that the cells expressed EMA and CEA, and did not express GFAP, S-100, vimentin (Vim) or TTF-1, which confirmed the endodermal origin of the cyst. The immunohistochemical staining for the Ki-67 proliferation marker was not expressed in any of the cells in the specimen (Fig. 3D). Based upon these findings, the pathological diagnosis of a typical $\mathrm{NC}$ without malignant transformation was made.

A histological examination of the tissue that was removed during the final craniotomy of the patient revealed well-formed papillae with prominent fibrovascular cores that were lined by malignant epithelial cells containing enlarged hyperchromatic nuclei with high nuclear-to-cytoplasmic ratios and nuclear abnormalities. Mitotic figures were easily observed (Fig. 4A and B). Frequent irregular folds of the dysplastic epithelial cells that showed a tendency to create well-formed papillae structures were observed in certain regions (Fig. 4C). The EMA and CEA immunohistochemical staining was more prominent in this final specimen compared with the prior surgical specimen. Immunohistochemical staining revealed that GFAP, Vim and TTF-1 were not expressed. The Ki-67 proliferative index had increased to $20-40 \%$ (Fig. 4D). The pathological diagnosis was of a well-differentiated papillary adenocarcinoma that originated from an initially benign NC. 


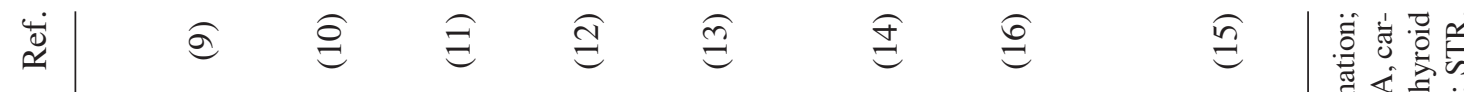

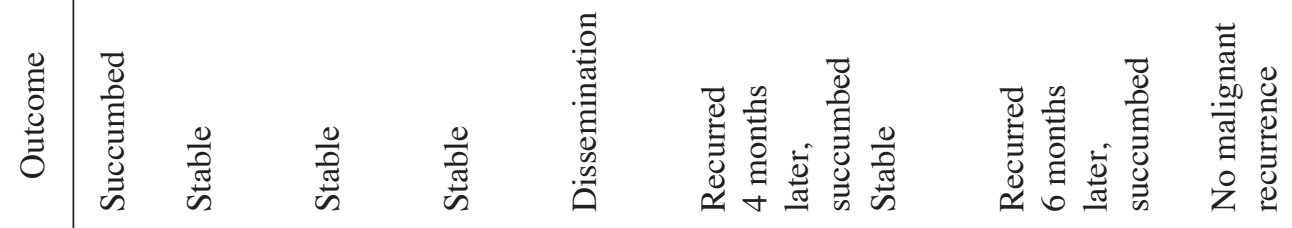

它咅

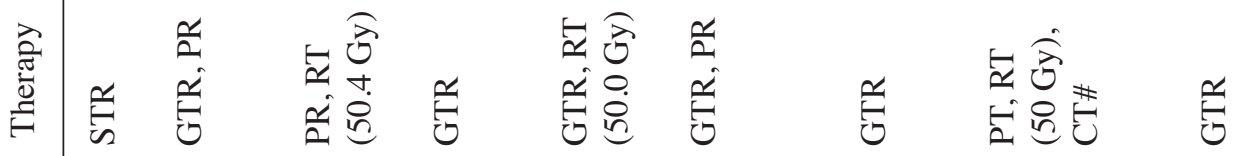

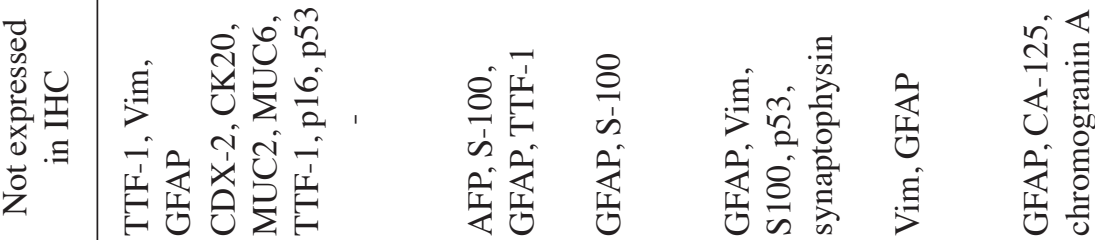





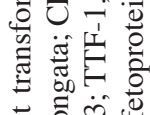

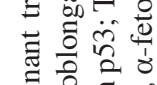

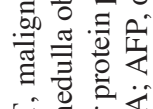

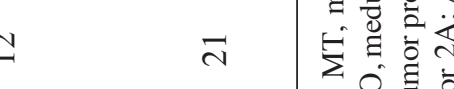

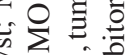

उें

:

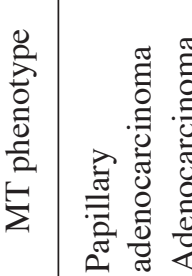

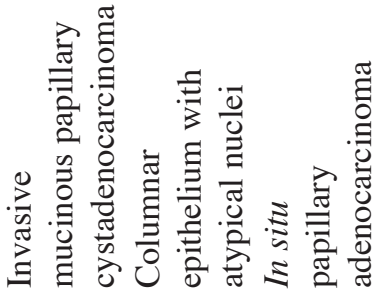

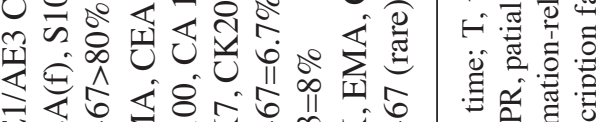

(ิ)

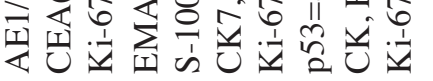

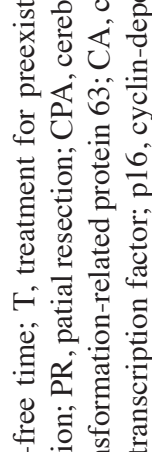

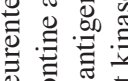

of

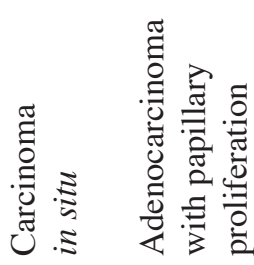

허웝

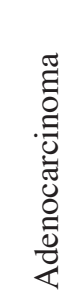

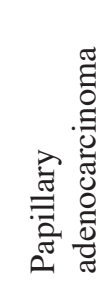

की

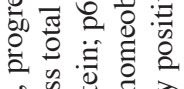

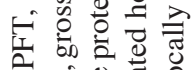

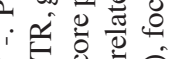

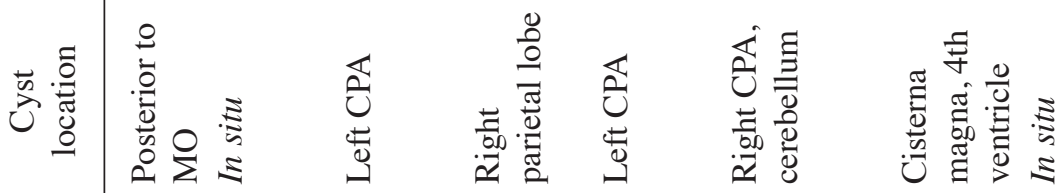

苛

o.

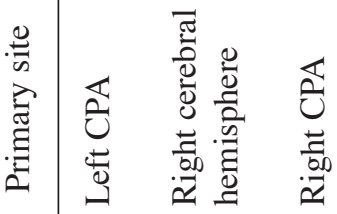

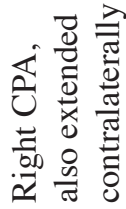

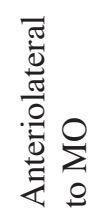



光 , 光

घ ते $\stackrel{\infty}{0}$

$\stackrel{x}{5} \simeq \simeq$

a 2 a $\frac{x}{0}$ a

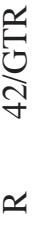

$\stackrel{\Xi}{\Xi}$

एँ

i.

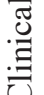

㱏

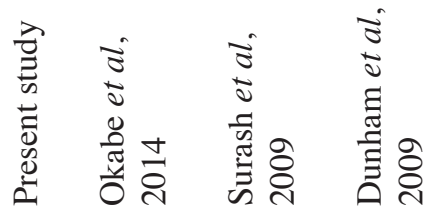

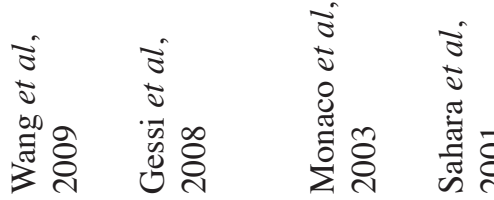






\section{Discussion}

NCs, also called enterogenous (13,15), enterogenic (17), endodermal (14) or archenteric (18) cysts, are congenital lesions of presumed ectodermal origin that rarely appear in the cranio-spinal axis. The precise pathogenesis of NCs remains unclear. The most popular hypothesis is that NCs develop from the failing separation between the foregut or the respiratory buds and the notochord during alimentary canal formation (1). A total surgical resection of the lesion is the most effective therapeutic method, as the residual cyst walls, particularly walls that are large enough to overlap, may cause recurrence $(1,6,8)$. Although recurrence and dissemination are not uncommon in NCs (19-21), the malignant transformation of $\mathrm{NC}$ is extremely rare.

Hamlat et al (22) and Bejjani et al (23) defined the criteria for the malignant transformation of intracranial epidermoid and dermoid cysts; however, no strict criteria for the malignant transformation of NCs have been previously developed. In order to determine the behavior of these lesions, previously published reports regarding intracranial and intraspinal malignant-transformed NCs were reviewed, following strict criteria for the selection of cases: The tumor must be restricted to the intracranial or intraspinal intradural compartment; the malignant transformation of a primary $\mathrm{NC}$ must have a benign $\mathrm{NC}$ wall component; and the malignant transformation in a recurrent $\mathrm{NC}$ must have preexisting pathologically confirmed NC. Metastasized carcinomas were excluded. Following these criteria, it was found that 8 cases (9-16) of malignantly transformed NCs have been described to date (Table I). In addition, malignant transformations were also identified in NCs outside of the cranio-spinal axis. Nakayama et al (24) reported a mucinous cyst adenocarcinoma in the sigmoid colon that was considered to originate from a NC.

Notably, all 9 studies, including the present study, were intracranial lesions, even though intraspinal NCs are more common compared with intracranial NCs. Intracranial NCs have been reported to comprise $\sim 10 \%$ of all NCs in the central nervous system $(3,8)$. Other reports have indicated that $\mathrm{NCs}$ are $\sim 3$ times more common in the spine compared with the brain (4); therefore, the lack of studies reporting an intraspinal $\mathrm{NC}$ with a malignant transformation is notable. In addition, in $3 / 9$ studies, the NC was located in the supratentorial region $(9,11,15)$, and 2 of those cases reported intramedullary lesions located in the cerebral hemisphere $(9,11)$. Intracranial NCs that developed within the posterior fossa accounted for $70-90 \%$ of all intracranial NC cases (1). Intramedullary supratentorial NCs appear to be much more rare. Despite the limited number of studies reviewed in the present study, the location distribution of NCs with malignant transformation did not coincide with the location distribution of all NCs. In addition, the time span of the 9 studies is hypothesized to have weakened this limitation.

The precise pathogenesis of the transformation and the original NCs remains unclear. Mittal et al (2) proposed that NCs are caused by anomalous endodermal cell migration that occurs dorsally through the primitive neurenteric canal into the ectoderm. This theory plausibly explains the progressively decreasing incidence of intraspinal, posterior fossa and supratentorial NCs. Based on this hypothesis, the ectopic endodermal cells that travelled the furthest distance may have been more likely to develop into dysplasia or a malignant transformation. This hypothesis may explain the variation in the location distribution between NCs with a malignant transformation and all NCs, acting as an internal cause. Other studies have suggested that chronic inflammation, most likely due to repeated cyst rupture or subtotal resection of the cyst walls, may predispose tissue to malignantly transform from benign epidermoid and dermoid cysts to squamous cell carcinomas $(22,25)$. A similar principle may apply to the development of malignantly transformed $\mathrm{NCs}$ and act as an external cause. These hypotheses are based on the existing limited cases and require additional studies in the future.

The male-to-female ratio of all 9 cases was 4:5, which did not indicate a significant difference between genders. The age of the 9 patients with $\mathrm{NC}$ and malignant transformation ranged between $25-60$ years $(6,9-14)$. To the best of our knowledge, no patients have been reported with malignantly transformed NC during childhood.

In 5 of the 9 patients (11-15), the malignant transformation occurred in the primary $\mathrm{NC}$, including in the 2 youngest patients $(12,13)$. The other 4 patients, including the patient from the present study, possessed benign primary NCs that transformed into malignant NCs upon recurrence $(9,10,12,16)$. The progression-free time between the benign primary NC and the malignant-transformed recurrent $\mathrm{NC}$ ranged from 22-168 months.

Intracranial NCs often appear with hyperintensity or isointensity on T1-weighted images and typical hyperintensity on T2-weighted images, without edema on MRI scans, and occasionally with rim enhancement or partial rim enhancement on Gd-enhanced MRI scans $(1,4)$. In the 9 studies reviewed in the present study, enhanced T1-weighted images were provided in 8 cases; of which, 6 cases, including the present case, showed enhancement $(9,10,11,15)$. Unlike the typical rim enhancement observed in benign NCs, the enhancement of NCs with malignant transformations was focal and heterogeneous. No direct evidence proves that these atypical enhanced regions are the transformed malignant component. Only 1 patient, who did not possess enhancement in the primary malignantly transformed $\mathrm{NC}$, possessed enhancement that was confirmed as tumor dissemination (12). In addition, 4 patients possessed multiloculated cysts on the MRI scans $(9,13,14,16)$, and 2 patients possessed part-cystic, part-solid structures on the MRI scans $(11,15)$. The lesion of the patient in the present study appeared as an enhanced solid mass that compressed the medulla oblongata. These radiological characteristics varied from those of the benign NCs, which appeared as a smooth cyst on the MRI scans. Additionally, surrounding edema, which is common with intracranial metastatic tumors, was identified in only 1 patient (16). For this patient, who possessed an extramedullary malignantly transformed $\mathrm{NC}$, the surrounding edema was local, which differs from the extensive edema of metastatic tumors. The exact radiological spectrum was not calculated due to the limited number of studies that were reviewed. However, adult primary or recurrent NCs with atypical radiological features, including multiloculated cysts, part-cystic and part-solid structures, or focal heterogeneous enhancement, are recommended to be assessed with the possibility of $\mathrm{NC}$ with 
malignant transformation in mind, and a relatively aggressive surgical strategy may be considered, as opposed to continued observation.

In the present literature review, the most common malignant pattern was adenocarcinoma on histological examination, which was identified in 6 patients, including the patient of the present case $(9,10,13,15,16)$. Papillary adenocarcinoma, characterized by distinct papillary architecture, was diagnosed in 4 patients, including the patient of the present case $(13,15,16)$. The other malignant patterns were papillary cystadenocarcinoma (11) and intraepithelial carcinoma (14). One intramedullary malignantly transformed NC was consistent with a papillary cyst adenocarcinoma with carcinomatous invasion of the brain parenchyma (11). Malignant epithelia accompanied with regional benign $\mathrm{NC}$ epithelia were present in 6 patients $(9,11-15)$. The other 3 patients, including the patient of the present case, did not possess benign epithelia in the transformed lesion $(10,16)$. Two cases were of carcinoma in situ $(14,16)$. Okabe et al (9) reported a mucinous adenocarcinoma with bronchopulmonary differentiation arising from the NC, which was almost the same as the pulmonary type I congenital adenomatoid malformation (CCAM); however, the $\mathrm{K}-\mathrm{R}$ as mutation and p-16 expression that frequently accompany CCAM were not detected.

Genetic analyses were not provided in the previous studies. This aspect of malignantly transformed NCs requires additional study, as comparing the gene expression in primary benign and recurrent malignantly transformed lesions may uncover the pathogenesis of malignantly transformed NCs. At present, no cases of the malignant transformation of $\mathrm{NCs}$ into squamous cell carcinoma have been observed. In the present study, the focal hyperplasia of epithelial cells prior to malignant transformation were identified. In addition, the frequent irregular folds of the dysplastic epithelium cells that showed a tendency to create a well-formed papillae structure were observed. These phenomena make up an intact process of malignant transformation, which supports our recommendation that $\mathrm{NC}$ with epithelial cells and focal hyperplasia requires careful examination and follow-up.

The Ki-67 labeling index of the malignantly transformed regions in the immunohistochemical examination ranged from rare to $80 \%$ expression $(9,10,12,14-16)$. Sahara et al (16) indicated that the staining of CEA and cancer antigen 19-9 in the recurrent malignantly transformed specimen was stronger compared with that in the initial benign specimen, and that the Ki-67 labeling index increased from $0 \%$ in the first surgical specimen to $6.7 \%$ in the second. In total, 3 patients possessed $\mathrm{Ki}-67$ labeling index scores of $>50 \%(9,12,14), 1$ of whom remained stable in the 2 years of follow-up. Wang et al (12) proposed that an increased Ki-67 labeling index score and the invasion of the tumor cells led to short recurrence and dissemination periods.

Surgery remains the major treatment for malignantly transformed and benign NCs. A total resection of the cyst content and cyst walls is recommended for a good prognosis (12). Since certain malignantly transformed NCs occur in recurrent $\mathrm{NCs}$, adhesion between the NC and surrounding structures may result in an incomplete resection $(10,16)$. In addition, primary malignant NCs may be adhered to surrounding tissues. Gessi et al (13) reported severe adhesion between a primary malignant NC and the surrounding structures, with mucinous cystic fluid. Monaco et al (14) described a primary malignantly transformed $\mathrm{NC}$ with clear, colorless and watery cystic fluid; however, adhesion between the cyst walls and surrounding tissues was not reported in the study. One primary intramedullary malignantly transformed NC with pathologically confirmed brain invasion was separated from the adjacent parenchymal white matter (11). These variable characteristics indicate that a recurrent malignantly transformed $\mathrm{NC}$ with mucinous fluid may adhere more easily to adjacent tissues. A previous study that reported the case of a benign intracranial $\mathrm{NC}$ indicated that the greater the size and the thinner the wall of the lesion, the stronger the adhesion to the surrounding tissue was (7). Due to the limited number of cases reviewed in the present study, this phenomenon cannot be confirmed for malignantly transformed NCs. The 2 of the 9 studies that reported the largest lesions did not mention severe adhesion between the lesion and surrounding tissues $(9,11)$. Malignant characteristics often indicate that adjuvant treatments, including radiotherapy $(11,16)$ and chemotherapy $(16)$, may be used. In a study regarding the malignant transformation of epidermoid and dermoid cysts, radiation therapy appeared to be beneficial, and the majority of patients that received radiation therapy survived for $>1$ year (22). Sahara et al (16) reported a case of malignantly transformed $\mathrm{NC}$ that was treated with a partial resection, 50 Gy radiotherapy and chemotherapy, including carboplatin and etoposide. The lesion recurred 6 months after surgery and eventually resulted in the mortality of the patient. Adjuvant treatment with radiotherapy was used in 2 other studies; 1 patient remained stable for 10 months after partial resection surgery (10), while in the other patient, the tumor disseminated 6 months after a total excision of the cyst (12).

In total, 4 patients from the 9 reviewed studies remained stable without disease progression or recurrence for the duration of the follow-up, which ranged from 3-38 months $(9,11,14,16)$. As the follow-up times were fairly short, additional follow-up examinations will be required for these 4 patients. Notably, 1 patient relapsed in $\sim 15$ months; however, the specimen from the second surgery showed fragments of the cyst wall that were identical to the benign component of the first specimen, without a malignant component or brain invasion (15). The exclusion of a malignant transformation of a recurrent lesion is challenging as the fenestration of the cyst wall of the second surgery may omit the malignant component. The remaining patients appeared to have poor prognoses. For 1 patient the tumor disseminated in 6 months (12), and 2 patients relapsed within 6 months and eventually succumbed to the disease $(13,16)$. The causes of the mortalities were associated with severe brainstem compression (13) and the extension of the tumor (16). In the present study, the patient's respiratory failure may have been due to brainstem compression and the infiltration of the dorsal medulla oblongata.

Overall, the malignant transformation of NCs in the central nervous system is extremely rare. Based on the findings of the present case and the review of the previous literature, strict criteria for malignant-transformed NCs can be defined. The location distribution of NCs with a malignant transformation does not coincide with the location distribution of all NCs. In general, the malignant transformation of NCs occurred in adult patients and demonstrated atypical radiological features. Therefore, an adult primary or recurrent NC with atypical radiological 
features may be more likely to become malignant. Surgery is the optimal treatment for malignantly transformed NCs, and close follow-up is required. The therapeutic effectiveness of adjuvant chemotherapy and radiotherapy requires additional study.

\section{References}

1. Gauden AJ, Khurana VG, Tsui AE and Kaye AH: Intracranial neuroenteric cysts: A concise review including an illustrative patient. J Clin Neurosci 19: 352-359, 2012.

2. Mittal S, Petrecca K, Sabbagh AJ, Rayes M, Melançon D, Guiot MC and Olivier A: Supratentorial neurenteric cysts - A fascinating entity of uncertain embryopathogenesis. Clin Neurol Neurosurg 112: 89-97, 2010.

3. Brooks BS, Duvall ER, el Gammal T, Garcia JH, Gupta KL and Kapila A: Neuroimaging features of neurenteric cysts: Analysis of nine cases and review of the literature. AJNR Am J Neuroradiol 14: 735-746, 1993.

4. Preece MT, Osborn AG, Chin SS and Smirniotopoulos JG: Intracranial neurenteric cysts: Imaging and pathology spectrum. AJNR Am J Neuroradiol 27: 1211-1216, 2006.

5. Simon JA, Olan WJ and Santi M: Intracranial neurenteric cysts: A differential diagnosis and review. Radiographics 17: 1587-1593, 1997.

6. Wang L, Zhang J, Wu Z, Jia G, Zhang L, Hao S and Geng S: Diagnosis and management of adult intracranial neurenteric cysts. Neurosurgery 68: 44-52, 2011.

7. de Oliveira RS, Cinalli G, Roujeau T, Sainte-Rose C, Pierre-Kahn A and Zerah M: Neurenteric cysts in children: 16 consecutive cases and review of the literature. J Neurosurg 103 (Suppl 6): S512-S523, 2005.

8. Al-Ahmed IH, Boughamoura M, Dirks P, Kulkarni AV, Rutka JT and Drake JM: Neurosurgical management of neurenteric cysts in children. J Neurosurg Pediatr 11: 511-517, 2013.

9. Okabe H, Katsura K, Yamano T, Tenjin H, Nakahara Y, Ishida M and Kato T: Mucinous adenocarcinoma arising from supratentorial intramedullary neuroenteric cyst with broncho-pulmonary differentiation. Neuropathology 34: 420-424, 2014.

10. Surash S, Ismail A, Loughrey C and van Hille P: Malignant transformation of a neurenteric cyst in the posterior fossa following complete excision. Br J Neurosurg 23: 458-461, 2009.

11. Dunham CP, Curry B and Hamilton M: Malignant transformation of an intraaxial-supratentorial neurenteric cyst - case report and review of the literature. Clin Neuropathol 28: 460-466, 2009.

12. Wang W, Piao YS, Gui QP, Zhang XH and Lu DH: Cerebellopontine angleneurenteric cyst with focal malignant features. Neuropathology 29: 91-95, 2009.
13. Gessi M, Legnani FG, Maderna E, Casali C, Solero CL, Pollo $\mathrm{B}$ and DiMeco F: Mucinous low-grade adenocarcinoma arising in an intracranial enterogenous cyst: Case report. Neurosurgery 62: E972-E973, 2008

14. Monaco R, Boscaino A, Di Blasi A, D’Antonio A, Profeta G, De Falco R and Nappi O: Intraepithelial carcinoma arising in an endodermal cyst of the posterior fossa. Neuropathology 23: 219-224, 2003

15. Ho LC, Olivi A, Cho CH, Burger PC, Simeone F and Tihan T: Well-differentiated papillary adenocarcinoma arising in a supratentorial enterogenous cyst: Case report. Neurosurgery 43: 1474-1477, 1998.

16. Sahara Y, Nagasaka T, Takayasu M, Takagi T, Hata N and Yoshida J: Recurrence of a neurenteric cyst with malignant transformation in the foramen magnum after total resection. Case report. J Neurosurg 95: 341-345, 2001.

17. Lara M, Pascual D, Aparicio MA, Ruiz L, Miranda D, Gomez-Moreta JA and Hernandez Vicente J: Giant and recurrent enterogenous cyst of the frontal lobe: Case report. Childs Nerv Syst 27: 1333-1339, 2011.

18. Hutchison $\mathbf{J}$ and Thomson JD: Congenital archenteric cysts. Br J Surg 41: 15-20, 1953.

19. Kimura H, Nagatomi A, Ochi M and Kurisu K: Intracranial neurenteric cyst with recurrence and extensive craniospinal dissemination. Acta Neurochir (Wien) 148: 347-352, 2006.

20. Chaynes P, Bousquet P, Sol JC, Delisle MB, Richaud J and Lagarrigue J: Recurrent intracranial neurenteric cysts. Acta Neurochir (Wien) 140: 905-911, 1998.

21. Perry A, Scheithauer BW, Zaias BW and Minassian HV: Aggressive enterogenous cyst with extensive craniospinal spread: Case report. Neurosurgery 44: 401-404, 404-405, 1999.

22. Hamlat A, Hua ZF, Saikali S, Laurent JF, Gedouin D, Ben-Hassel $M$ and Guegan Y: Malignant transformation of intra-cranial epithelial cysts: Systematic article review. J Neurooncol 74: 187-194, 2005.

23. Bejjani GK, Wright DC, Schessel D and Sekhar LN: Endodermal cysts of the posterior fossa. Report of three cases and review of the literature. J Neurosurg 89: 326-335, 1998.

24. Nakayama H, Akikusa B, Kondo Y, Saito N, Sarashina H and Okui K: Mucinous cystadenocarcinoma of the colon. Report of a case. Dis Colon Rectum 32: 243-246, 1989.

25. Abramson RC, Morawetz RB and Schlitt M: Multiple complications from an intracranial epidermoid cyst: Case report and literature review. Neurosurgery 24: 574-578, 1989. 